\title{
Neural Correlations Between Executive Function and Social Function in Autism Spectrum Disorder: A Mediation Analysis of fMRI
}

\section{Anyi Zhang}

Peking University Sixth Hospital https://orcid.org/0000-0003-0673-9554

\section{Le Shi}

Peking University Sixth Hospital

Xiao Lin

Peking University Sixth Hospital

\section{Suhua Chang}

Peking University Sixth Hospital

Peng Li

Peking University Sixth Hospital

\section{Yanping Bao}

Peking University

Wei Yan

Peking University Sixth Hospital

\section{Li Yang}

Peking University Sixth Hospital

Jie Shi

Peking University

\section{Jiahui Deng}

Peking University Sixth Hospital

Lin Lu

Peking University Sixth Hospital

Jiajia Liu ( $\square$ liujiajia_sdu@bjmu.edu.cn )

Peking University https://orcid.org/0000-0001-5548-8777

\section{Research}

Keywords: autism spectrum disorder, social function, executive function, ABIDEIl, fMRI, functional connectivity.

Posted Date: August 30th, 2021

DOI: https://doi.org/10.21203/rs.3.rs-838445/v1

License: () (i) This work is licensed under a Creative Commons Attribution 4.0 International License. Read Full License 


\section{Abstract}

Background: Autism spectrum disorder (ASD) is a set of neurodevelopmental disorders with high heterogeneity. The co-occurrence of social deficits and executive dysfunction is frequently reported in individuals with ASD. The present study evaluated the association between social deficit and executive dysfunction in ASD subjects and explored the underlying neural mechanisms that may mediate this association.

Methods: A total of 186 patients with ASD, 5-18 years old (10.25 \pm 2.72 years old), from the Autism Brain Imaging Data Exchange II database were enrolled in the final sample. Social function was evaluated by the parent-reported Social Responsiveness Scale (SRS), and executive function (EF) was measured by the parent-reported Behavior Rating Inventory of Executive Function (BRIEF). We selected bilateral amygdala, caudate and putamen as regions of interests, and used the region of interest-based resting-state functional connectivity (FC) analysis to explore the shared and specific brain mechanisms underlying EF and social function. The association analysis of EF, social deficits and FC was conducted using linear regression by controlling covariates, including age, gender, full IQ, handedness, and current medication.

Results: The result showed that social functions in ASD children and adolescents were positively associated with EF $(r=0.612, p$ $<0.01$ ). Additionally, 14 and $21 \mathrm{FC}$ links were associated with social deficits and executive dysfunction separately, in which five FC links (right amygdala and right inferior frontal gyrus opercular part, left caudate and right supramarginal gyrus, left putamen and right superior frontal gyrus medial orbital, right putamen and left gyrus rectus, and right putamen and left paracentral lobule) were simultaneously correlated with social function and EF in ASD subjects. We also found that connectivity between the right amygdala and right inferior frontal gyrus mediated the effects of EF on social deficit.

Limitations: All the included participants in the present study were ASD patients and male participants, which might limit the generalization of our findings.

Conclusion: We found a significant association between social deficits and executive dysfunction in ASD subjects. This association might be mediated by FC between the right amygdala and the right inferior frontal gyrus, which may further illustrate the underlying mechanism of ASD and co-occurrence of social deficits and executive dysfunction in this population.

\section{Background}

Autism spectrum disorder (ASD) is a set of neurodevelopmental disorders that is characterized by an early-onset of social deficits, restricted interests and repetitive behaviors(1). Previously, children with ASD were always diagnosed at the age of 3-4 years, while, the atypical development of social function might be identified at 6-12 months old(1). The prevalence of ASD has increased from $0.01 \%$ in 1931 to nearly $2 \%$ in $2020(2,3)$. Social function is essential for development of relationships and fitness to the environment and emerges gradually and dynamically through childhood and adolescence(4). As we live in a largely social constructed world, the social interaction with others was very important due to its contributions for survival and reproductive fitness(5). While, the inappropriate or absence of social behaviors in ASD patients resulted in significant disability. Disruptions of social skills and behaviors might be related to psychological stress, low self-esteem, and deficits in executive function $(E F)(4,6)$. In addition to the core clinical characteristics in ASDs, deficit in EF was also described as a prevalence feature(7). Executive function comprises a set of higher cognitive functions that mediate goal-directed activities(8). The cognitive components that are involved in EF include inhibition, emotional control, flexibility, working memory, set shifting, planning, and organization(9). Studies previously described that deficit in EF might affect the mental and physical health, school life and employment, cognitive development and social process(10). Evidence has shown that executive dysfunction might contribute to the dysfunction of other main characteristics of ASD individual, including social interaction, adaptive behavior, and school performance(11-14).

ASD patients might have difficulty inhibiting information that would interrupt efficient social and cognitive behavior $(15,16)$. Moreover, EF was shown to impact adaptation and internalization in patients with ASD(6). ASD patients with typica social deficits often fail to adjust their behavior to the environment, which might be explained by executive dysfunction, thereby impacting many aspects across the lifespan including reading ability in childhood, resilience in adolescents, and social interaction in adulthood(17-19). Moreover, emotional control is an important ability in social communication. ASD individuals with executive dysfunction might present impairments in emotional control, which further contributes to social deficits(20). It has also been

Page 2/19 
reported that improvement of EF might be a possible intervention target for the improvement in social functions in ASDs(6). The underlying mechanism that explains assessing this relationship, however, remains unclear.

Advances in brain imaging researches have helped us understand the neural mechanisms for many disorders, including ASD. In ASD, abnormalities that are associated with social deficits were mainly located in the "social brain," including the amygdala, insula, inferior frontal gyrus, posterior superior temporal sulcus, temporo-parietal junction and dorsomedial prefrontal cortex $(21,22)$. According to a previous meta-analysis summarizing the fMRI characteristics of 655 ASD children, amygdala impairment was reported as a core abnormality in ASD patients that might contribute to social deficit, and dysfunctions in facial recognition(23). Additionally, neural-imaging evidences have suggested that executive dysfunction in ASD individuals might originate from the atypical activation and connectivity of the insular, cerebellum, right middle temporal cortex $(24,25)$. Aberrant striatal function has been reported in autistic brain, as the two important components: caudate and putamen were involved in many cognitive processes (rewarding, reinforcement learning etc.) social interaction and stereotype behaviors(26). One of the potential targets for ASD patients reported in the previous study were stratum, which was functionally related to the social and rewarding(27). As a result, the neuro-imaging provided a crucial way to identify the underlying neural mechanism that mediated the effect of EF on social function in patients with ASD. Few studies have evaluated the underlying neural mechanisms that mediate the effect of EF on social function in patients with ASD, thus leaving a pressing need to better understand the neural bases of this association.

Moreover, there are limited pharmacological treatment for ASD patients, it is urgent to expand the intervention options and potential brain targets(27). In the present study, we (1) evaluated the association between executive dysfunction and social deficits in ASD patients and (2) identified the underlying neural mechanisms for this association by performing a regions-of-interests (ROIs)-based resting-state functional connectivity (FC) analysis to better inform potential interventions and therapeutic targets.

\section{Method}

\section{Participants}

Participants were recruited from the Autism Brain Imaging Data Exchange II (ABIDEII) database, which was established to further promote scientific discovery of the brain connectome in $\operatorname{ASD}(28)$. The ABIDEIl database is publicly available and consists of data from 19 international sites, that share phenotypic characteristics, anatomical imaging, and resting-state functional magnetic resonance imaging (fMRI) of a total of 521 ASD patients and 593 healthy controls(28). The inclusion criteria for this study were the following: (1) patients diagnosed with ASD using the Diagnostic and Statistical Manual of Mental Disorders, 5th Edition, and confirmed by the Autism Diagnostic Observation Schedule (ADOS) and the Autism Diagnostic Interview, Revised (ADI-R), (2) ASD patients who were assessed for the social function using the Social Responsiveness Scale (SRS), and (3) ASD patients who were assessed for EF using the Behavior Rating Inventory of Executive Function (BRIEF). Participants were excluded if they lacked the following data: gender, age, handedness, full intelligence quotient (IQ), SRS score, or BRIEF score. Moreover, subjects were excluded if they presented excessive head motions (beyond $2 \mathrm{~mm}$ translation or $2^{\circ}$ rotation) during the scan. A total of 186 patients with ASD, $5-18$ years old ( $10.25 \pm 2.72$ years old), were included in this study, which came from five datasets: Georgetown University, San Diego State University, New York University Langone Medical Center (samples 1 and 2), and Kennedy Krieger Institute.

\section{Measures}

Social Responsiveness Scale. The parent-reported SRS is a widely used measure to screen social function in ASD individuals aged 4-18 years old(29). Previous studies found that SRS scores were highly related to ADI-R scores(30, 31). The SRS is a 65-item 4point Likert scale, from 0 (never true) to 3 (almost true). The SRS total score ranges from 0 to 195. The scale measures five clusters of social function: social awareness, social cognition, social communication, social motivation and autistic mannerisms(32). We used the SRS total T-score in the mediation analyses. According to Bruni, SRS T-score higher than 76 was considered as severe impairment in social function(32).

Behavior Rating Inventory of Executive Function. Executive function was measured using the parent-reported BRIEF. The BRIEF has 86 items on a 3-point Likert scale, ranging from 1 (never) to 3 (often). The BRIEF is helpful for understanding children's behaviors in different environments(33). The BRIEF consists of two broader indices (Behavioral Regulation Index [BRI] and Metacognition Index $[\mathrm{MI}]$ ), and eight subscales (inhibition, shift, emotional control, initiate, working memory, plan/organize, and organization of 
materials). The T-score of Global Executive Composite (GEC) was used in the mediation analyses. Patients with a T score $\geq 65$ represented clinically significant EF problems, and higher scores meant poorer EF(34).

Full IQ. The tests that were used to assess full IQ included Different Ability Scale-2nd edition (DAS-II), Wechsler Abbreviated Scale of Intelligence (WASI), Wechsler Abbreviated Scale of Intelligence-2nd edition (WASI-II), and Wechsler Intelligence Scale of Children - 4th and 5th editions (WISC-IV, WISC-V). Consistency among these IQ tests was identified in previous studies $(35,36)$.

Handedness. Handedness categories were right-handed, left-handed and mixed-handed. All sites in the ABIDEIl database noted the handedness of every participant, and four sites collected handedness strength scores(28). Coding for the scores was $\geq 50$ (righthanded), $\leq-50$ (left-handed), $-50 \sim 50$ (mixed-handed)(28).

\section{Magnetic resonance imaging data preprocessing}

Regions-of-interests selection. In our study, we selected bilateral amygdala, putamen and caudate as the core ROls. According to a previous meta-analysis, core brain abnormalities in ASD patients were the amygdala, caudate and putamen, based on resting-state fMRI(37). Studies previously reported that the amygdala is an important hub in many cognitive processes (e.g., emotion, social, reward, etc.)(38, 39). Extensive evidence also showed that amygdala abnormalities in ASD affect social behavior (40), Therefore, we chose the amygdala as an ROI. Additionally, the caudate and putamen are two key components of the striatum. Striatum abnormalities correlated with the severity of social deficits in ASD patients, and the striatum has been implicated in ASD pathogenesis(41). Thus, the caudate and putamen were also included as ROls. Altogether, the six summarized ROls were left amygdala, right amygdala, left caudate, right caudate, left putamen and right putamen, which were selected and the coordinate were extracted from the Anatomical Automatic Labeling (AAL) atlas $(67 \times 71 \times 67)(42)$.

Imaging Preprocessing. We extracted the resting-state fMRI of each patients for further analysis. The detailed sequence parameters were shown in TableS1 (available online). Resting-state fMRI data preprocessing was conducted using the Data Processing and Analysis for (Resting-State) Brain Imaging (DPABI) toolbox in MATLAB(43). The first four volumes of each subject were removed, and the remaining volumes were then slice-timing corrected, head motion realigned (participants with excessive head motion were excluded), spatially normalized to standard Montreal Neurological Institute (MNI) stereotaxic space, resampled to $3 \times 3 \times 3 \mathrm{~mm}^{3}$, smoothened with a $6 \mathrm{~mm}$ full-width at half-maximum (FWHM) Gaussian kernel and bandpass filtered $(0.01 \mathrm{~Hz}-$ $0.1 \mathrm{~Hz})$.

FC measure. Next, we calculated the ROls-based resting-state FC in all participants using the DPABI toolbox as well. Whole brain FC was calculated by correlating the time series of the ROls and the rest brain regions in AAL 90 atlas, and we extracted the $z$ transformed FC to confirm normality (6×89 brain regions with 534 edges for all subjects) using the DPABI toolbox.

\section{Statistical analysis}

We firstly analyzed the correlation of executive dysfunction and social deficit in ASD children with ASD using the Pearson correlation analysis with Bonferroni correction after controlling covariates, including age, gender, full IQ, and current medication. Next, whether FC correlated with executive dysfunction and social deficits separately. Brain regions that were related to social deficits and executive dysfunction were assessed using Pearson correlation analysis. A General Linear Model (GLM) that controlled covariates (i.e., gender, full IQ, handedness, and current medication [0 for not currently taking medication, 1 for currently taking medication]) were analyzed to further verify the correlation. To address multiple comparisons, we also controlled the False Discovery Rate (FDR). The FCs with $p<0.05$ (FDR corrected) were considered as significant.

We also identified shared FC links that simultaneously correlated with EF and social function in ASD patients. We applied model 4 with 5,000 bias-corrected bootstrap resamples in PROCESS to explore whether the shared FC links significantly mediated the association between executive dysfunction (independent variable) and social deficits (output variable) in ASD children(44). The 95\% confidence interval (Cl) didn't contain 0 was considered as significant in the mediation analysis.

\section{Results}

\section{Demographic characteristics}


The final participants in our study included 186 ASD children, aged $5-18$ years old (mean $=10.25$ years, standard deviation [SD] = 2.73 years), from five datasets. Most of them were boys ( $n=154,82.8 \%$ ). The participants' full IQs ranged from 63 to 149 (mean = $107.54, S D=17.32)$. The children in our study were mostly right-handed $(78 \%, 145$ of 186$)$. The prevalence of severe social deficits was $50.5 \%$ (94 of 186) among the participants. The prevalence of executive dysfunction was $66.7 \%$ (124 of 186; Table 1).

Table 1

Demographic characteristics of the participants $(n=186)$

\begin{tabular}{|c|c|c|c|}
\hline \multirow[t]{2}{*}{ characteristics } & \multicolumn{3}{|c|}{ ASD $(n=186)$} \\
\hline & Mean /n & SD/proportion (\%) & Range \\
\hline Age (years) & 10.25 & 2.73 & $5.13-18.00$ \\
\hline Sex (girls) & 32 & $17.2 \%$ & NA \\
\hline Full IQ & 107.54 & 17.32 & $63-149$ \\
\hline Handedness (right-handed) & 145 & $78.0 \%$ & NA \\
\hline SRS_social awaness_T & 69.91 & 13.02 & $39-98$ \\
\hline SRS_social cognition_T & 73.11 & 13.71 & $39-108$ \\
\hline SRS_social communication_T & 75.04 & 13.67 & $39-110$ \\
\hline SRS_social motivation_T & 71.17 & 14.03 & $4-113$ \\
\hline SRS_autistic mannerisms_T & 79.61 & 16.11 & $40-129$ \\
\hline SRS_total_T & 77.40 & 13.42 & $42-116$ \\
\hline Severe social deficit & 94 & $50.5 \%$ & NA \\
\hline BRIEF_inhibition_T & 62.21 & 12.48 & $36-97$ \\
\hline BRIEF_shift_T & 68.61 & 13.01 & $37-99$ \\
\hline BRIEF_emotional control_T & 60.99 & 12.22 & $37-91$ \\
\hline BRIEF_BRI_T & 65.39 & 11.66 & $34-99$ \\
\hline BRIEF_initiate_T & 64.23 & 10.95 & $36-89$ \\
\hline BRIEF_working memory_T & 66.14 & 11.19 & $35-90$ \\
\hline BRIEF_plan/organization_T & 65.24 & 11.48 & $33-89$ \\
\hline BRIEF_organization of material_T & 58.65 & 10.41 & $33-72$ \\
\hline BRIEF_monitor_T & 64.24 & 10.99 & $34-88$ \\
\hline BRIEF_MI_T & 66.17 & 10.42 & $37-86$ \\
\hline BRIEF_GEC_T & 79.61 & 16.11 & $40-129$ \\
\hline Executive dysfunction & 124 & $66.7 \%$ & NA \\
\hline
\end{tabular}

\section{Correlation between social function and EF in ASD}

After controlling covariates including age, gender, full IQ, and current medication, social functions in ASD children were positively associated with the EF. Higher scores on the BRIEF BRI, MI, and GEC in children with ASD were related to higher SRS scores. As shown in Table 2, a positive relationship was found between almost all subscales of the SRS and BRIEF. However, correlations 
between social motivation and inhibition, social cognition, social communication, social motivation and autistic mannerism with organization were not significant.

Table 2

Correlations between social deficits and executive dysfunction in ASD children $(n=186)$.

\begin{tabular}{|c|c|c|c|c|c|c|}
\hline & $\begin{array}{l}\text { SRS_social } \\
\text { awaness_T }\end{array}$ & $\begin{array}{l}\text { SRS_social } \\
\text { cognition_T }\end{array}$ & $\begin{array}{l}\text { SRS_social } \\
\text { communication_T }\end{array}$ & $\begin{array}{l}\text { SRS_social } \\
\text { motivation_T }\end{array}$ & $\begin{array}{l}\text { SRS_autistic } \\
\text { mannerisms_T }\end{array}$ & $\begin{array}{l}\text { SRS_total } \\
-T\end{array}$ \\
\hline BRIEF_inhibition_T & $0.423^{\star}$ & $0.315^{\star}$ & $0.395^{\star}$ & 0.221 & $0.390^{\star}$ & $0.396^{\star}$ \\
\hline BRIEF_shift_T & $0.432^{\star}$ & $0.542^{\star}$ & $0.559 *$ & $0.511^{\star}$ & $0.620^{\star}$ & $0.633^{*}$ \\
\hline BRIEF_emotional control_T & 0.277 & $0.481^{\star}$ & $0.426^{*}$ & $0.487^{*}$ & $0.447^{\star}$ & $0.503^{*}$ \\
\hline BRIEF_BRI_T & $0.465^{\star}$ & $0.544^{*}$ & $0.561^{*}$ & $0.490^{\star}$ & $0.589 *$ & $0.622^{*}$ \\
\hline BRIEF_initiate_T & $0.338^{*}$ & $0.359 *$ & $0.430 *$ & $0.456^{\star}$ & $0.359 *$ & $0.463^{*}$ \\
\hline BRIEF_working memory_T & $0.382^{\star}$ & $0.361^{\star}$ & $0.404^{*}$ & $0.363^{\star}$ & $0.346^{\star}$ & $0.436^{\star}$ \\
\hline BRIEF_plan/organization_T & $0.424^{\star}$ & $0.370 *$ & $0.364^{*}$ & $0.290 *$ & $0.305^{\star}$ & $0.405^{\star}$ \\
\hline $\begin{array}{l}\text { BRIEF_organization of } \\
\text { material_T }\end{array}$ & $0.361^{*}$ & 0.168 & 0.219 & 0.138 & 0.154 & 0.221 \\
\hline BRIEF_monitor_T & $0.537^{\star}$ & $0.411^{*}$ & $0.505^{\star}$ & $0.290 *$ & $0.414^{\star}$ & $0.515^{\star}$ \\
\hline BRIEF_MI_T & $0.511^{\star}$ & $0.426^{*}$ & $0.483^{*}$ & $0.383^{\star}$ & $0.394^{*}$ & $0.513^{*}$ \\
\hline BRIEF_GEC_T & $0.540 *$ & $0.518^{*}$ & $0.563^{*}$ & $0.473^{*}$ & $0.525^{\star}$ & $0.612^{*}$ \\
\hline
\end{tabular}

Functional connectivity links associated with social deficits

Fourteen FC links were associated with social function, evaluated by the SRS, in ASD children (Table 3, Fig. 1). The Pearson correlation analysis results were consistent with the results of the General Linear Models. We totally found fourteen FC links associated with social function in ASD individuals, including nine links for positive association that means children with an increased FC in those links would presented with a higher level of social deficit, and five links for negative association that means the increased FC in those links were mostly founded in children with lower level of social deficit. Details were shown in Table 3 and Fig. 1. 
Table 3

Functional connectivity links correlated with SRS total T scores in ASD children $(n=186)$.

\begin{tabular}{|c|c|c|c|}
\hline Seed regions & Effect regions & $r(p, p F D R c o r n)$ & $\beta(p, p F D R c o r r)$ \\
\hline \multirow[t]{3}{*}{ Amygdala_L } & Insula_R & $0.172(0.021,0.032)$ & $2.418(0.021,0.032)$ \\
\hline & Cingulum_Ant_R & $-0.169(0.023,0.032)$ & $-2.918(0.023,0.032)$ \\
\hline & Parietal_Inf_L & $-0.187(0.012,0.032)$ & $-1.239(0.012,0.032)$ \\
\hline Amygdala_R & Frontal_Inf_Oper_R & $0.302(<0.001,<0.001)$ & $2.757(<0.001,0.005)$ \\
\hline \multirow[t]{2}{*}{ Caudate_L } & SupraMarginal_R & $0.231(0.002,0.014)$ & $1.467(0.002,0.014)$ \\
\hline & SupraMarginal_L & $0.158(0.034,0.037)$ & $1.519(0.034,0.037)$ \\
\hline \multirow[t]{2}{*}{ Caudate_R } & Amygdala_L & $-0.185(0.013,0.032)$ & $-1.886(0.013,0.032)$ \\
\hline & SupraMarginal_L & $0.155(0.038,0.038)$ & $1.163(0.037,0.037)$ \\
\hline \multirow[t]{2}{*}{ Putamen_L } & Frontal_Mid_Orb_R & $-0.175(0.019,0.032)$ & $-1.357(0.019,0.032)$ \\
\hline & Calcarine_R & $0.165(0.027,0.033)$ & $2.836(0.025,0.032)$ \\
\hline \multirow[t]{4}{*}{ Putamen_R } & Rectus_L & $-0.170(0.023,0.032)$ & $-1.078(0.022,0.032)$ \\
\hline & Hippocampus_R & $0.203(0.007,0.032)$ & $7.057(0.008,0.032)$ \\
\hline & Parietal_Sup_L & $0.172(0.022,0.032)$ & $1.642(0.022,0.032)$ \\
\hline & Paracentral_Lobule_L & $0.164(0.028,0.033)$ & $1.623(0.029,0.034)$ \\
\hline \multicolumn{4}{|c|}{$\begin{array}{l}\text { SRS, Social Responsiveness Scale; ASD, autism spectrum disorder; L, left; R, right; Ant, anterior; Inf, inferior; Oper, opercular; Mi } \\
\text { middle; Orb, orbital; Sup, superior. pfdrcorr. FDR-corrected } p \text { value. }\end{array}$} \\
\hline
\end{tabular}

\section{Functional connectivity links associated with executive dysfunction}

In the correlation analysis, we found that executive dysfunction in patients with ASD was associated with alterations of FC between 27 brain regions and 21 FC links after controlling covariates. As shown in Table 4 and Fig. 2, more effect regions were located at left hemisphere. In the Pearson correlation analysis, there were eleven FC links negatively related to the executive dysfunction in ASD individuals which means that children with an increased connectivity in those links would present a lower risk for executive function. While, there were thirteen FC links negatively related to the executive dysfunction in the GLM. In addition, the rest eight FC links were positively related to the executive dysfunction, that the increased connectivity might contribute to a decreased executive function, and higher risk for executive dysfunction. Details were shown in Table 4 and Fig. 2. 
Table 4

Functional connectivity links correlated with BRIEF global executive composite T scores in ASD children $(n=186)$.

\begin{tabular}{|c|c|c|c|}
\hline Seed region & Effect region & $r(p, p F D R c o r r)$ & $\beta(p, p F D R c o r r)$ \\
\hline \multirow[t]{2}{*}{ Amygdala_L } & Angular_R & $-0.164(0.029,0.034)$ & $-0.901(0.031,0.041)$ \\
\hline & Thalamus_L & $-0.165(0.028,0.034)$ & $-2.420(0.046,0.046)$ \\
\hline \multirow[t]{5}{*}{ Amygdala_R } & Frontal_Sup_L & $-0.166(0.027,0.034)$ & $-0.827(0.045,0.046)$ \\
\hline & Frontal_Mid_R & $-0.149(0.046,0.046)$ & $-0.706(0.045,0.046)$ \\
\hline & Frontal_Inf_Oper_R & $0.251(0.001,0.019)$ & $1.794(0.001,0.021)$ \\
\hline & Occipital_Sup_L & $0.197(0.008,0.032)$ & $1.002(0.008,0.041)$ \\
\hline & Temporal_Inf_L & $-0.158(0.025,0.034)$ & $-1.234(0.024,0.041)$ \\
\hline \multirow[t]{3}{*}{ Caudate_L } & Rolandic_Oper_L & $-0.190(0.011,0.032)$ & $-1.405(0.013,0.041)$ \\
\hline & SupraMarginal_R & $0.197(0.008,0.032)$ & $0.973(0.008,0.041)$ \\
\hline & Heschl_L & $-0.164(0.011,0.032)$ & $-1.297(0.029,0.041)$ \\
\hline \multirow[t]{3}{*}{ Caudate_R } & Frontal_Mid_R & $-0.161(0.031,0.035)$ & $-1.053(0.031,0.041)$ \\
\hline & Rectus_L & $0.168(0.024,0.032)$ & $0.885(0.024,0.041)$ \\
\hline & Cingulum_Post_R & $0.150(0.045,0.046)$ & $2.650(0.045,0.046)$ \\
\hline \multirow[t]{3}{*}{ Putamen_L } & Frontal_Sup_L & $-0.171(0.023,0.034)$ & $-1.140(0.031,0.041)$ \\
\hline & Frontal_Mid_Orb_R & $-0.180(0.016,0.032)$ & $-0.979(0.031,0.041)$ \\
\hline & Temporal_Mid_L & $0.192(0.010,0.032)$ & $1.417(0.016,0.041)$ \\
\hline \multirow[t]{5}{*}{ Putamen_R } & Frontal_Sup_Orb_L & Not significant & $-0.780(0.027,0.041)$ \\
\hline & Frontal_Mid_L & $0.183(0.016,0.032)$ & $1.230(0.015,0.041)$ \\
\hline & Rolandic_Oper_L & Not significant & $-1.607(0.046,0.046)$ \\
\hline & Rectus_L & $-0.187(0.014,0.032)$ & $-0.887(0.016,0.041)$ \\
\hline & Paracentral_Lobule_L & $0.182(0.017,0.032)$ & $1.589(0.006,0.041)$ \\
\hline
\end{tabular}

\section{Overlapping FC links for the association between social deficit and executive dysfunction and mediation analysis}

According to the correlation analysis, the shared FC links that were associated with SRS scores and BRIEF scores simultaneously in patients with ASD included the right amygdala and right inferior frontal gyrus opercular part, left caudate and right supramarginal gyrus, left putamen and right superior frontal gyrus medial orbital, right putamen and left gyrus rectus, and right putamen and left paracentral lobule (Fig. 3). We then assessed whether these five FC links significantly mediated the effect of executive dysfunction on social deficits in patients with ASD using mediation analysis. We found one FC link that mediated the association between SRS scores and BRIEF scores. As shown in Fig. 4, connectivity between the right amygdala and right inferior frontal gyrus mediated the effects of BRIEF scores on SRS scores. The mediation effects of other FC links are shown in Fig. S1 (available online).

\section{Discussion}


The present study explored the association between social function and EF in children with ASD and found that the social deficit was positively associated with the executive dysfunction. We further identified the shared underlying brain FC link between right amygdala and right inferior frontal gyrus might mediate the effect of EF on social function. These findings provided a new insight for understanding the neuro mechanisms of the co-occurrence of social deficit and executive dysfunction in ASD patients, and further inform the intervention target.

The present results confirmed that social deficits and executive dysfunction were positively related to each other in children with ASD, and social deficits in ASD children were predominately accompanied by executive dysfunction. These results were similar to previous findings, which indicated that components of EF might contribute to social communication in $\operatorname{ASD}$ patients $(6,14,45,46)$. The two major components of EF were positively related to the social awareness, social cognition, and social communication in the present study. The association between organization of material in EF and social cognition, communication, motivation, autistic mannerisms, emotional control in EF and social awareness in social deficit, inhibition in EF and social motivation in social function were not statistically significant. As described in a previous study, the organization of materials domain was not significantly different between children with ASD and typically developing children (47). As a result, abnormalities in the organization of materials might not be a predictor of ASD or social function in children with ASD. Studies that evaluated inhibitory control in ASD children reported mixed results. A review reported that inhibitory control ability in ASD would predict future scholastic, occupational, and social interaction performance(10). Further studies are needed to clarify the association between inhibition and autistic mannerisms. The shift domain reflects the ability to change from one situation to another, and it was closely related to higher-order restricted and repetitive behaviors and interests(48). In the present study, we did not find a statistically significant correlation between shift and autistic mannerisms.

The present results indicated that brain regions that are related to social deficits in children with ASD were mostly located in the supramarginal gyrus, which is also involved in visual experiences(49). The supramarginal gyrus and angular gyrus are two components of the inferior parietal cortex, which is an important hub in the default mode network (50). Alterations of activation of the supramarginal gyrus, inferior partial cortex, and default mode network were commonly reported in children with ASD $(51,52)$. Alterations of FC between the supramarginal gyrus and caudate were previously reported to be involved in other cognition processes, including hyperarousal symptoms in insomnia patients and secondary language vocabulary acquisition $(53,54)$. In our study, it might also be related to social function in ASD children. Moreover, the supramarginal gyrus might be implicated in failure of the emotional self-other distinction in children with $\operatorname{ASD}(55)$. Numerous imaging studies suggested that FC abnormalities in the supramarginal gyrus are related to emotional processes, such as interoception, empathy, and attentional processing(55-57).

Social-emotional dysfunctions are core symptoms in patients with ASD. Interventions or training that target emotional recognition and understanding might in turn impact social interaction in $\operatorname{ASD}$ patients $(20,58)$. Other brain regions in the present study that were related to social function in ASD children included the frontal lobe and limbic lobe. Previous studies reported that the inferior frontal gyrus was abnormally activated in ASD children during emotional-social expression $(59,60)$. Atypical FC in the limbic lobe and cingulate was also observed in ASD patients(61). The cingulate is an important component of the limbic lobe. It is a white matter joint of the frontal lobe, parietal lobe, and temporal lobe, and this brain region is related to EF, emotion, and pain(62). The hippocampus is another brain region in the limbic lobe that is related to memory and relational recoding(63). Changes in FC between the limbic lobe and amygdala and between the limbic lobe and putamen in the present study were consistent with a previous study that showed that social deficits in ASD patients might be related to atypical amygdala-cingulate connectivity(64).

Executive dysfunction is a common symptom in ASD patients. Brain regions that are associated with impairments in EF were predominantly located in the frontal lobe, including the superior part, inferior part, and middle part. Several studies provided evidence that the frontal lobe is a key brain region for EF. Damage to the frontal lobe might contribute to executive dysfunction in many disorders(65-68). The frontal lobe is involved various cognitive and emotional processes. Developmental changes in frontal lobe function occur during aging, which can impact executive control, decision-making, working memory, and inhibition(69). Individuals with ASD might exhibit the prolonged heterogeneity of frontal lobe dysfunction(70). In the present study, abnormal FC links were found between the frontal lobe and amygdala, caudate, and putamen in ASD children. Consistent with a previous study, alterations of frontal-striatal connectivity contribute to deficits in facial viewing, which is another core characteristic of children with ASD(71). The left gyrus rectus is a part of the inferior frontal gyrus (IFG) and involved in social interaction(72). This region was significantly associated with EF in children with ASD in the present study and previous studies $(72,73)$. Correlations were found between the right amygdala and left superior occipital gyrus and between the left caudate and supramarginal gyrus in ASD 
children who presented executive dysfunction, which was previously reported in autistic subjects $(74,75)$. According to Simard et al., the occipital gyrus is related to visual and cognitive processing, such as the identification of correct answers in a reasoning $\operatorname{task}(74)$.

Functional connectivity links that significantly mediated the association between social deficits and executive dysfunction included the right amygdala and right inferior frontal gyrus. The amygdala and inferior frontal gyrus were reported to play a role in social and emotional processes $(76,77)$. Atypical frontal lobe development and function have been described in many psychological disorders. The frontal lobe is involved in many cognitive progresses, including emotion, personality, self-awareness, social function, and attention(78). Abnormal frontal lobe-amygdala connectivity has been previously reported in ASD patients, which could contribute to social deficits(79). The atypical activation of these brain regions would prevent appropriate reactions to social or emotional stimuli (76). The results of a previous neuroimaging meta-analysis suggested that subjects with ASD exhibited aberrant activation in the bilateral inferior frontal gyrus and bilateral amygdala during face processing(80). Executive function was associated with the amygdala, the dorsolateral prefrontal cortex, and connectivity between core regions, including the amygdala and thalamus(81). Accumulating evidence suggests changes in activation of the amygdala and inferior frontal gyrus in ASD subjects and associations between FC and clinical characteristics. The present study found that alterations of FC between these two regions mediated the correlation between two characteristics of ASD-social deficits and executive dysfunction-which might reveal a neural mechanism of the pathogenesis of ASD. Furthermore, our findings focused on the behavioral associated amygdalacircuits, which might expand the treatment routes for ASD subjects.

The present study has several limitations. First, all participants were ASD subjects. The co-occurrence of social deficits and executive dysfunction can also be observed in the general population of children. Unknown is whether the mediation effect we found is also appliable to typically developing children with social deficits and executive dysfunction. Second, we used various evaluations of social function and EF in children with ASD. We chose the SRS and BRIEF because correlations between SRS and BRIEF scores have been widely described in the literature. Further studies that evaluate these associations using other measurements are needed. Third, the majority of the participants were boys. The prevalence of autism is $4-5$ times higher in males than in females.

In conclusion, we found a significant association between social deficits and executive dysfunction in ASD children. We also explored the mediation effect of FC links and found that alterations of FC between the amygdala and inferior frontal gyrus significantly mediated the correlation between clinical symptoms, which may further illustrate the underlying mechanism of ASD.

\section{Abbreviations}

AAL

Anatomical Automatic Labeling

ABIDEII

Autism Brain Imaging Data Exchange II

ADI-R

Autism Diagnostic Interview, Revised

ADOS

Autism Diagnostic Observation Schedule (ADOS)

ASD

Autism spectrum disorder

BRIEF

Behavior Rating Inventory of Executive Function

BRI

Behavioral Regulation Index

DAS-II

Different Ability Scale-2nd edition

DPABI

Data Processing and Analysis for (Resting-State) Brain Imaging

Page 10/19 
executive function

FC

functional connectivity

FDR

False Discovery Rate

fMRI

functional magnetic resonance imaging

FWHM

full-width at half-maximum

GEC

Global Executive Composite

IFG

inferior frontal gyrus

MI

Metacognition Index

$\mathrm{MNI}$

Montreal Neurological Institute

ROI

regions-of-interest

SRS

Social Responsiveness Scale.

WASI

Wechsler Abbreviated Scale of Intelligence

WASI-II

Wechsler Abbreviated Scale of Intelligence-2nd edition

WISC-IV

Wechsler Intelligence Scale of Children-4th editions

WISC-V

Wechsler Intelligence Scale of Children-5th editions

\section{Declarations}

Ethics approval and consent to participant

Data included in our study were applied and extracted from the open-source database, all participants and their caregivers have signed the consent at every dataset.

\section{Consent for publication}

Not applicable.

Availability of data and materials

All data used and analyzed in the current study are available from the open-source database, which is available at the website http://fcon_1000.projects.nitrc.org/indi/abide/abide_Il.html.

Competing interests

All authors declare that they have no competing interests.

Funding 
This work was supported in part by the National Natural Science Foundation of China (no. 81821092, and 81761128036), National Key Research and Development Program of China (no. 2019YFA0706200), Fundamental Research Funds of the Central Universities (no. 69004Y1230), Beijing Municipal Science and Technology Commission (Z181100001518005), PKU-Baidu Fund (2020BD011).

\section{Authors' contributions}

AZ wrote the initial draft of manuscript with contributions from $\mathrm{JL}$ and $\mathrm{LL}$. AZ and JL developed the study design and analysis plan. $A Z$ and $J L$ conducted the data analyses with help from $L S, X L, S C, J D, P L, Y B$. $L S, X L, S C, J D, P L, Y B$ JL, and $L L$ critically revised the manuscript. All coauthors approved the results and final version.

\section{Acknowledgements}

All authors would thank all the participants and all the researcher in universities that make contributions to the database.

\section{References}

1. Lai MC, Lombardo MV, Baron-Cohen S. Autism Lancet. 2014;383(9920):896-910.

2. Nevison C, Blaxill M, Zahorodny W. California autism prevalence trends from 1931 to 2014 and comparison to national ASD data from IDEA and ADDM. J Autism Dev Disord. 2018;48(12):4103-17.

3. Maenner MJ, Shaw KA, Baio J, Washington A, Patrick M, DiRienzo M, et al. Prevalence of autism spectrum disorder among children aged 8 years - autism and developmental disabilities monitoring network, 11 sites, United States, 2016. MMWR Surveill Summ. 2020;69(4):1-12.

4. Beauchamp MH, Anderson V. SOCIAL: an integrative framework for the development of social skills. Psychol Bull. 2010;136(1):39-64.

5. Chen P, Hong W. Neural Circuit Mechanisms of Social Behavior. Neuron. 2018;98(1):16-30.

6. Bednarz HM, Trapani JA, Kana RK. Metacognition and behavioral regulation predict distinct aspects of social functioning in autism spectrum disorder. Child Neuropsychol. 2020;26(7):953-81.

7. Demetriou EA, Lampit A, Quintana DS, Naismith SL, Song YJC, Pye JE, et al. Autism spectrum disorders: a meta-analysis of executive function. Mol Psychiatry. 2018;23(5):1198-204.

8. Zhang Z, Peng P, Zhang D. Executive function in high-functioning autism spectrum disorder: a meta-analysis of fMRI studies. J Autism Dev Disord. 2020;50(11):4022-38.

9. Hill EL. Executive dysfunction in autism. Trends Cogn Sci. 2004;8(1):26-32.

10. Diamond A. Executive functions. Annu Rev Psychol. 2013;64:135-68.

11. Chouinard B, Gallagher L, Kelly C. He said, she said: Autism spectrum diagnosis and gender differentially affect relationships between executive functions and social communication. Autism. 2019;23(7):1793-804.

12. Freeman LM, Locke J, Rotheram-Fuller E, Mandell D. Brief report: examining executive and social functioning in elementaryaged children with autism. J Autism Dev Disord. 2017;47(6):1890-5.

13. Van Eylen L, Boets B, Steyaert J, Wagemans J, Noens I. Executive functioning in autism spectrum disorders: influence of task and sample characteristics and relation to symptom severity. Eur Child Adolesc Psychiatry. 2015;24(11):1399-417.

14. Fong VC, larocci G. The role of executive functioning in predicting social competence in children with and without autism spectrum disorder. Autism Res. 2020;13(11):1856-66.

15. Christ SE, Holt DD, White DA, Green L. Inhibitory control in children with autism spectrum disorder. J Autism Dev Disord. 2007;37(6):1155-65.

16. Dempster FN. The rise and fall of the inhibitory mechanism: toward a unified theory of cognitive development and aging. Dev Rev. 1992;12(1):45-75.

17. Dajani DR, Uddin LQ. Demystifying cognitive flexibility: Implications for clinical and developmental neuroscience. Trends Neurosci. 2015;38(9):571-8. 
18. Engel de Abreu PM, Abreu N, Nikaedo CC, Puglisi ML, Tourinho CJ, Miranda MC, et al. Executive functioning and reading achievement in school: a study of Brazilian children assessed by their teachers as "poor readers". Front Psychol. 2014;5:550.

19. Genet JJ, Siemer M. Flexible control in processing affective and non-affective material predicts individual differences in trait resilience. Cogn Emot. 2011;25(2):380-8.

20. Høyland AL, Nærland T, Engstrøm M, Lydersen S, Andreassen OA. The relation between face-emotion recognition and social function in adolescents with autism spectrum disorders: A case control study. PLoS One. 2017;12(10):e0186124.

21. Gotts SJ, Simmons WK, Milbury LA, Wallace GL, Cox RW, Martin A. Fractionation of social brain circuits in autism spectrum disorders. Brain. 2012;135(Pt 9):2711-25.

22. Moessnang C, Baumeister S, Tillmann J, Goyard D, Charman T, Ambrosino S, et al. Social brain activation during mentalizing in a large autism cohort: the Longitudinal European Autism Project. Mol Autism. 2020;11(1):17.

23. Costa C, Cristea IA, Dal Bò E, Melloni C, Gentili C. Brain activity during facial processing in autism spectrum disorder: an activation likelihood estimation (ALE) meta-analysis of neuroimaging studies. J Child Psychol Psychiatry. 2021.

24. Wichers RH, Findon JL, Jelsma A, Giampietro V, Stoencheva V, Robertson DM, et al. Modulation of atypical brain activation during executive functioning in autism: a pharmacological MRI study of tianeptine. Mol Autism. 2021;12(1):14.

25. Nomi JS, Molnar-Szakacs I, Uddin LQ. Insular function in autism: Update and future directions in neuroimaging and interventions. Prog Neuropsychopharmacol Biol Psychiatry. 2019;89:412-26.

26. Kuo HY, Liu FC. Pathological alterations in striatal compartments in the human brain of autism spectrum disorder. Mol Brain. 2020;13(1):83.

27. Pretzsch CM, Floris DL, Voinescu B, Elsahib M, Mendez MA, Wichers R, et al. Modulation of striatal functional connectivity differences in adults with and without autism spectrum disorder in a single-dose randomized trial of cannabidivarin. Mol Autism. 2021;12(1):49.

28. Di Martino A, O'Connor D, Chen B, Alaerts K, Anderson JS, Assaf M, et al. Enhancing studies of the connectome in autism using the autism brain imaging data exchange II. Sci Data. 2017;4:170010.

29. Constantino JN. Social Responsiveness Scale. Encyclopedia of Autism Spectrum Disorders; 2013.

30. Li C, Zhou H, Wang T, Long S, Du X, Xu X, et al. Performance of the autism spectrum rating scale and social responsiveness scale in identifying autism spectrum disorder among cases of intellectual disability. Neurosci Bull. 2018;34(6):972-80.

31. Constantino JN, Davis SA, Todd RD, Schindler MK, Gross MM, Brophy SL, et al. Validation of a brief quantitative measure of autistic traits: comparison of the social responsiveness scale with the autism diagnostic interview-revised. J Autism Dev Disord. 2003;33(4):427-33.

32. Bruni TP. Test review: social responsiveness scale-second edition (SRS-2). J Psychoeduc Assess. 2014;32(4):365-9.

33. Peters C, Algina J, Smith SW, Daunic AP. Factorial validity of the Behavior Rating Inventory of Executive Function (BRIEF)Teacher form. Child Neuropsychol. 2012;18(2):168-81.

34. McLean RL, Johnson Harrison A, Zimak E, Joseph RM, Morrow EM. Executive function in probands with autism with average IQ and their unaffected first-degree relatives. J Am Acad Child Adolesc Psychiatry. 2014;53(9):1001-9.

35. Kuriakose S. Concurrent validity of the WISC-IV and DAS-II in children with autism spectrum disorder. J Psychoeduc Assess. 2014;32(4):283-94.

36. Scott WC, Austin DW, Reid DS. Investigation of the WISC-III and WASI in clinical child populations: a framework for further exploration. Can J of Sch Psychol. 2007;22(2):249-54.

37. Zhang A, Shi L, Yuan K, Lin X, Han Y, Bao Y, Shi J, Liu J, Lu L. Large-scale brain network dysfunction in autism spectrum disorder: a meta-analysis of resting-state fMRI. (In preparation). 2021.

38. Hennessey T, Andari E, Rainnie DG. RDoC-based categorization of amygdala functions and its implications in autism. Neurosci Biobehav Rev. 2018;90:115-29.

39. Rutishauser U, Mamelak AN, Adolphs R. The primate amygdala in social perception - insights from electrophysiological recordings and stimulation. Trends Neurosci. 2015;38(5):295-306.

40. Baron-Cohen S, Ring HA, Bullmore ET, Wheelwright S, Ashwin C, Williams SC. The amygdala theory of autism. Neurosci Biobehav Rev. 2000;24(3):355-64.

Page 13/19 
41. Langen M, Schnack HG, Nederveen H, Bos D, Lahuis BE, de Jonge MV, et al. Changes in the developmental trajectories of striatum in autism. Biol Psychiatry. 2009;66(4):327-33.

42. Tzourio-Mazoyer N, Landeau B, Papathanassiou D, Crivello F, Etard O, Delcroix N, et al. Automated anatomical labeling of activations in SPM using a macroscopic anatomical parcellation of the MNI MRI single-subject brain. Neuroimage. 2002;15(1):273-89.

43. Yan CG, Wang XD, Zuo XN, Zang YF. DPABI: Data processing \& analysis for (resting-state) brain imaging. Neuroinformatics. 2016;14(3):339-51.

44. Hayes A. Introduction to mediation, moderation, and conditional process analysis. J Educ Meas. 2013;51(3):335-7.

45. Lai CLE, Lau Z, Lui SSY, Lok E, Tam V, Chan Q, et al. Meta-analysis of neuropsychological measures of executive functioning in children and adolescents with high-functioning autism spectrum disorder. Autism Res. 2017;10(5):911-39.

46. Joseph RM, Tager-Flusberg $\mathrm{H}$. The relationship of theory of mind and executive functions to symptom type and severity in children with autism. Dev Psychopathol. 2004;16(1):137-55.

47. Gardiner E, larocci G. Everyday executive function predicts adaptive and internalizing behavior among children with and without autism spectrum disorder. Autism Res. 2018;11(2):284-95.

48. Faja S, Nelson Darling L. Variation in restricted and repetitive behaviors and interests relates to inhibitory control and shifting in children with autism spectrum disorder. Autism. 2019;23(5):1262-72.

49. Mahon BZ, Schwarzbach J, Caramazza A. The representation of tools in left parietal cortex is independent of visual experience. Psychol Sci. 2010;21(6):764-71.

50. Abbott AE, Nair A, Keown CL, Datko M, Jahedi A, Fishman I, et al. Patterns of atypical functional connectivity and behavioral links in autism differ between default, salience, and executive networks. Cereb Cortex. 2016;26(10):4034-45.

51. Solomon M, Ragland JD, Niendam TA, Lesh TA, Beck JS, Matter JC, et al. Atypical learning in autism spectrum disorders: A functional magnetic resonance imaging study of transitive inference. J Am Acad Child Adolesc Psychiatry. 2015;54(11):94755.

52. Salmi J, Roine U, Glerean E, Lahnakoski J, Nieminen-von Wendt T, Tani P, et al. The brains of high functioning autistic individuals do not synchronize with those of others. Neuroimage Clin. 2013;3:489-97.

53. Lee YG, Kim S, Kim N, Choi JW, Park J, Kim SJ, et al. Changes in subcortical resting-state functional connectivity in patients with psychophysiological insomnia after cognitive-behavioral therapy: Changes in resting-state FC after CBT for insomnia patients. Neuroimage Clin. 2018;17:115-23.

54. Hosoda C, Tanaka K, Nariai T, Honda M, Hanakawa T. Dynamic neural network reorganization associated with second language vocabulary acquisition: a multimodal imaging study. J Neurosci. 2013;33(34):13663-72.

55. Hoffmann F, Koehne S, Steinbeis N, Dziobek I, Singer T. Preserved self-other distinction during empathy in autism islinked to network integrity of right supramarginal gyrus. J Autism Dev Disord. 2016;46(2):637-48.

56. Lamm C, Decety J, Singer T. Meta-analytic evidence for common and distinct neural networks associated with directly experienced pain and empathy for pain. Neuroimage. 2011;54(3):2492-502.

57. Carter RM, Huettel SA. A nexus model of the temporal-parietal junction. Trends Cogn Sci. 2013;17(7):328-36.

58. Carver LJ, Dawson G. Development and neural bases of face recognition in autism. Mol Psychiatry. 2002;7(Suppl 2):18-20.

59. Bastiaansen JA, Thioux M, Nanetti L, van der Gaag C, Ketelaars C, Minderaa R, et al. Age-related increase in inferior frontal gyrus activity and social functioning in autism spectrum disorder. Biol Psychiatry. 2011;69(9):832-8.

60. Dapretto M, Davies MS, Pfeifer JH, Scott AA, Sigman M, Bookheimer SY, et al. Understanding emotions in others: mirror neuron dysfunction in children with autism spectrum disorders. Nat Neurosci. 2006;9(1):28-30.

61. Lau WKW, Leung MK, Zhang R. Hypofunctional connectivity between the posterior cingulate cortex and ventromedial prefrontal cortex in autism: Evidence from coordinate-based imaging meta-analysis. Prog Neuropsychopharmacol Biol Psychiatry. 2020;103:109986.

62. Bubb EJ, Metzler-Baddeley C, Aggleton JP. The cingulum bundle: Anatomy, function, and dysfunction. Neurosci Biobehav Rev. 2018;92:104-27. 
63. Cooper RA, Richter FR, Bays PM, Plaisted-Grant KC, Baron-Cohen S, Simons JS. Reduced hippocampal functional connectivity during episodic memory retrieval in autism. Cereb Cortex. 2017;27(2):888-902.

64. Velasquez F, Wiggins JL, Mattson WI, Martin DM, Lord C, Monk CS. The influence of 5-HTTLPR transporter genotype on amygdala-subgenual anterior cingulate cortex connectivity in autism spectrum disorder. Dev Cogn Neurosci. 2017;24:12-20.

65. Stuss DT. Functions of the frontal lobes: relation to executive functions. J Int Neuropsychol Soc. 2011;17(5):759-65.

66. Koechlin E. An evolutionary computational theory of prefrontal executive function in decision-making. Philos Trans R Soc Lond B Biol Sci. 2014;369(1655).

67. Dirlikov B, Shiels Rosch K, Crocetti D, Denckla MB, Mahone EM, Mostofsky SH. Distinct frontal lobe morphology in girls and boys with ADHD. Neuroimage Clin. 2015;7:222-9.

68. Pope RA, Thompson PJ, Rantell K, Stretton J, Wright MA, Foong J. Frontal lobe dysfunction as a predictor of depression and anxiety following temporal lobe epilepsy surgery. Epilepsy Res. 2019;152:59-66.

69. Rosso IM, Young AD, Femia LA, Yurgelun-Todd DA. Cognitive and emotional components of frontal lobe functioning in childhood and adolescence. Ann N Y Acad Sci. 2004;1021:355-62.

70. Uddin LQ. Brain mechanisms supporting flexible cognition and behavior in adolescents with autism spectrum disorder. Biol Psychiatry. 2021;89(2):172-83.

71. Sims TB, Neufeld J, Johnstone T, Chakrabarti B. Autistic traits modulate frontostriatal connectivity during processing of rewarding faces. Soc Cogn Affect Neurosci. 2014;9(12):2010-6.

72. Bi XA, Zhao J, Xu Q, Sun Q, Wang Z. Abnormal functional connectivity of resting state network detection based on linear ICA analysis in autism spectrum disorder. Front Physiol. 2018;9:475.

73. Xu S, Li M, Yang C, Fang X, Ye M, Wei L, et al. Altered functional connectivity in children with low-function autism spectrum disorders. Front Neurosci. 2019;13:806.

74. Simard I, Luck D, Mottron L, Zeffiro TA, Soulières I. Autistic fluid intelligence: Increased reliance on visual functional connectivity with diminished modulation of coupling by task difficulty. Neuroimage Clin. 2015;9:467-78.

75. Aoki S, Kagitani-Shimono K, Matsuzaki J, Hanaie R, Nakanishi M, Tominaga K, et al. Lesser suppression of response to bright visual stimuli and visual abnormality in children with autism spectrum disorder: a magnetoencephalographic study. J Neurodev Disord. 2019;11(1):9.

76. Rudie JD, Shehzad Z, Hernandez LM, Colich NL, Bookheimer SY, lacoboni M, et al. Reduced functional integration and segregation of distributed neural systems underlying social and emotional information processing in autism spectrum disorders. Cereb Cortex. 2012;22(5):1025-37.

77. Di Martino A, Ross K, Uddin LQ, Sklar AB, Castellanos FX, Milham MP. Functional brain correlates of social and nonsocial processes in autism spectrum disorders: an activation likelihood estimation meta-analysis. Biol Psychiatry. 2009;65(1):63-74.

78. Chayer C, Freedman M. Frontal lobe functions. Curr Neurol Neurosci Rep. 2001;1(6):547-52.

79. Swartz JR, Wiggins JL, Carrasco M, Lord C, Monk CS. Amygdala habituation and prefrontal functional connectivity in youth with autism spectrum disorders. J Am Acad Child Adolesc Psychiatry. 2013;52(1):84-93.

80. Nickl-Jockschat T, Rottschy C, Thommes J, Schneider F, Laird AR, Fox PT, et al. Neural networks related to dysfunctional face processing in autism spectrum disorder. Brain Struct Funct. 2015;220(4):2355-71.

81. Rohr CS, Dreyer FR, Aderka IM, Margulies DS, Frisch S, Villringer A, et al. Individual differences in common factors of emotional traits and executive functions predict functional connectivity of the amygdala. Neuroimage. 2015;120:154-63.

\section{Figures}




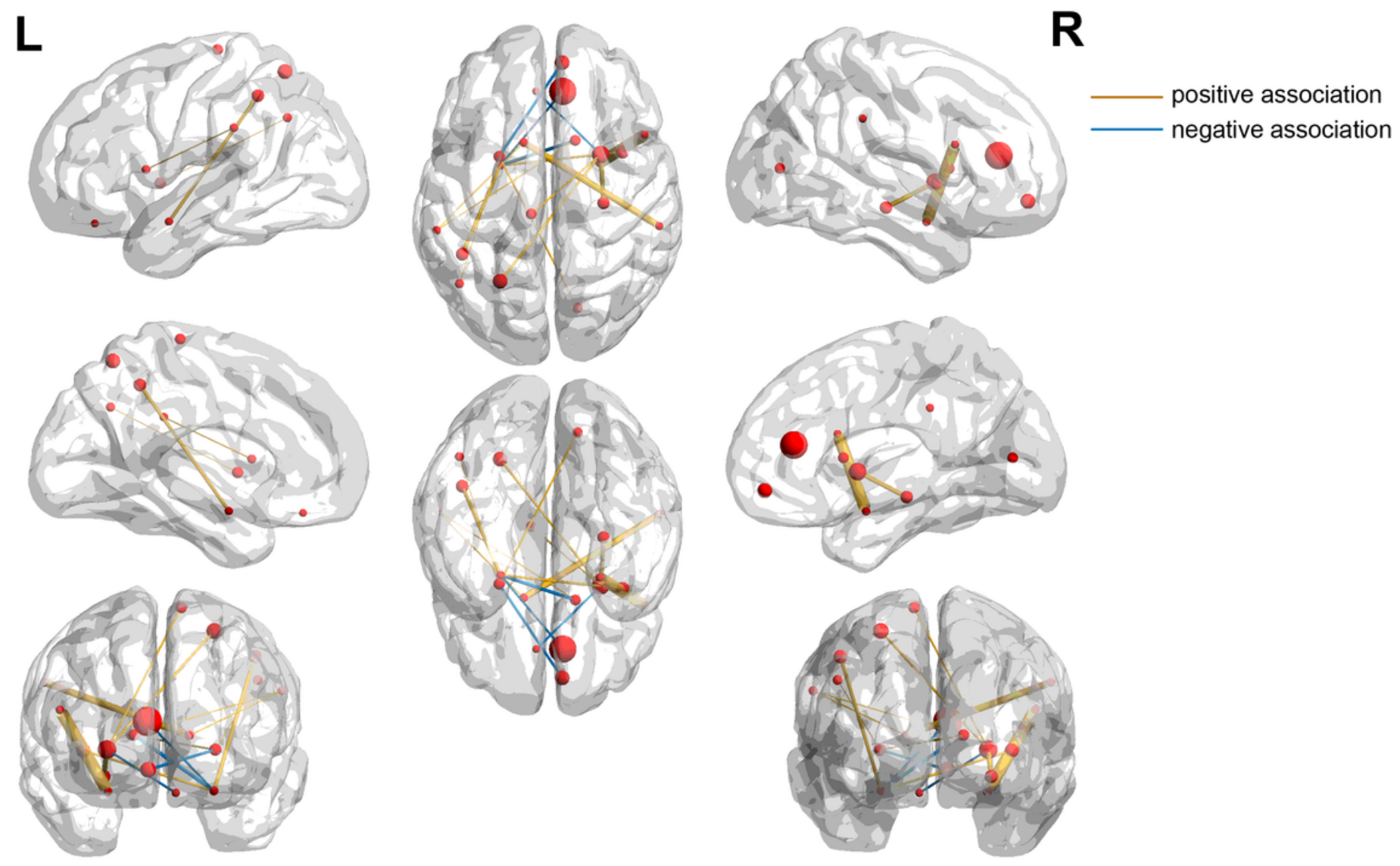

Figure 1

Functional connectivity links associated with SRS scores. The yellow edges are positively related to SRS scores. The blue edges are negatively related to SRS scores. 
$\mathbf{L}$
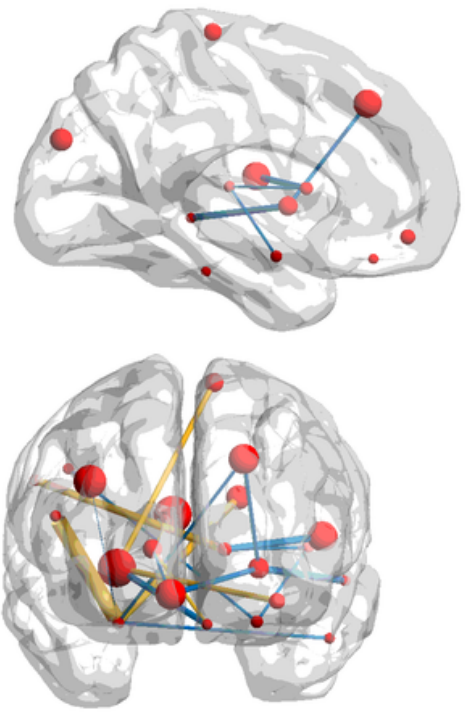
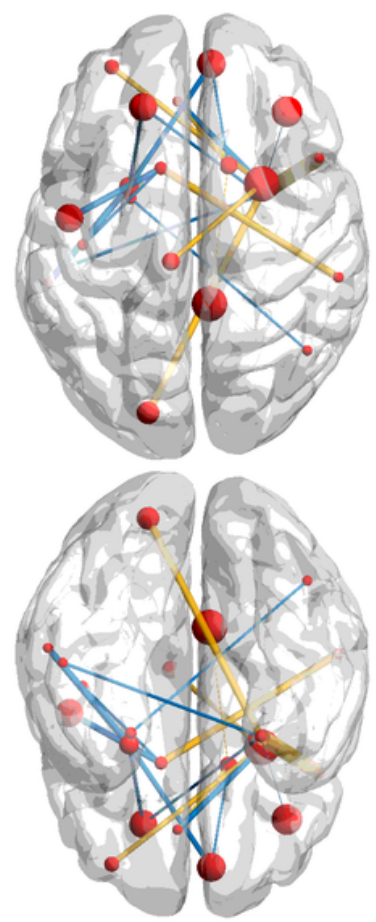

$\mathbf{R}$
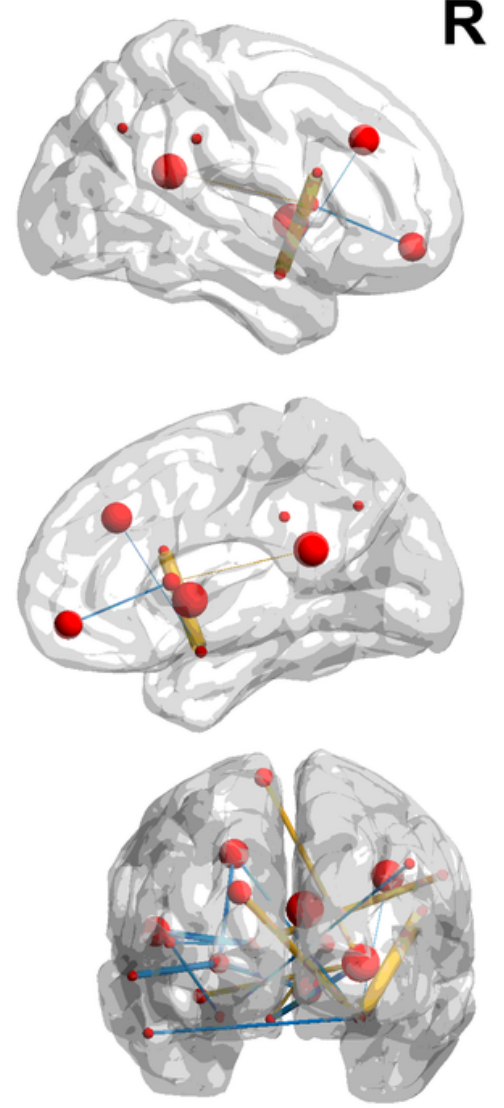

positive association

negative association

\section{Figure 2}

Functional connectivity links associated with BRIEF scores. The yellow edges are positively related to BRIEF scores. The blue edges are negatively related to BRIEF scores. 

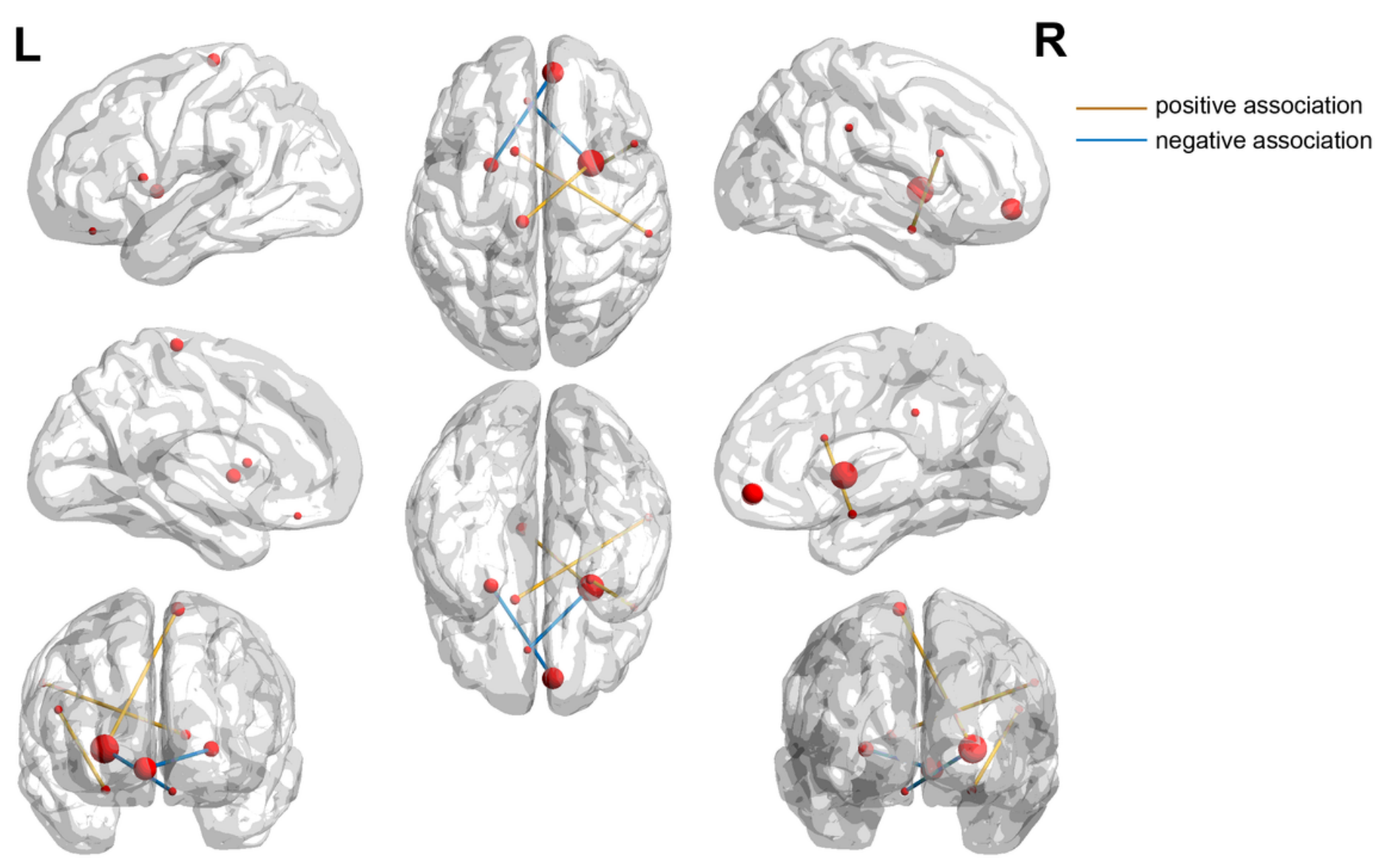

Figure 3

Shared FC links between SRS scores and BRIEF scores. The yellow edges are positively related to both SRS scores and BRIEF scores. The blue edges are negatively related to both SRS scores and BRIEF scores.

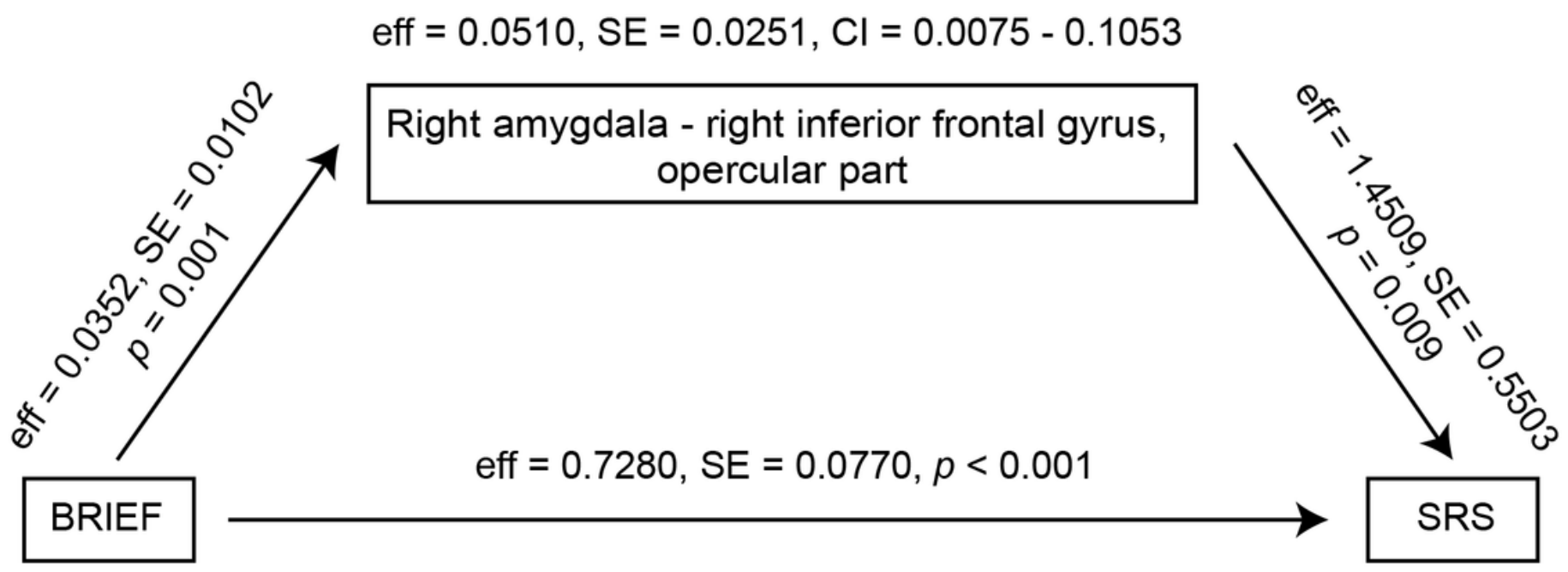

Figure 4

Mediation effect of shared FC links for the association between SRS scores and BRIEF scores. SRS, Social Responsiveness Scale; BRIEF, Behavior Rating Inventory of Executive Function; eff, effect; SE, standard error; Cl, confidence interval.

\section{Supplementary Files}


This is a list of supplementary files associated with this preprint. Click to download.

- additionalfile1.docx 